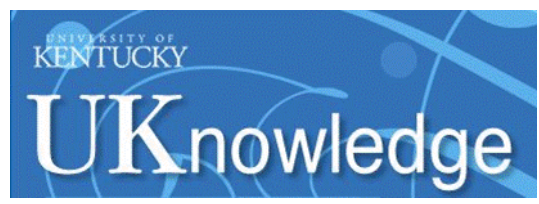

University of Kentucky

UKnowledge

3-16-2018

\title{
Experimental Examination of Vorticity Stripping from a Wing-Tip Vortex in Free-Stream Turbulence
}

Hari C. Ghimire

University of Kentucky, hari.ghimire@uky.edu

Sean C. C. Bailey

University of Kentucky, sean.bailey@uky.edu

Follow this and additional works at: https://uknowledge.uky.edu/me_facpub

Part of the Fluid Dynamics Commons, and the Mechanical Engineering Commons

Right click to open a feedback form in a new tab to let us know how this document benefits you.

\section{Repository Citation}

Ghimire, Hari C. and Bailey, Sean C. C., "Experimental Examination of Vorticity Stripping from a Wing-Tip Vortex in Free-Stream Turbulence" (2018). Mechanical Engineering Faculty Publications. 43.

https://uknowledge.uky.edu/me_facpub/43

This Article is brought to you for free and open access by the Mechanical Engineering at UKnowledge. It has been accepted for inclusion in Mechanical Engineering Faculty Publications by an authorized administrator of UKnowledge. For more information, please contact UKnowledge@lsv.uky.edu. 


\section{Experimental Examination of Vorticity Stripping from a Wing-Tip Vortex in Free- Stream Turbulence}

Digital Object Identifier (DOI)

https://doi.org/10.1103/PhysRevFluids.3.034702

Notes/Citation Information

Published in Physical Review Fluids, v. 3, issue 3, 034702, p. 1-11.

C2018 American Physical Society

The copyright holder has granted the permission for posting the article here. 


\title{
Experimental examination of vorticity stripping from a wing-tip vortex in free-stream turbulence
}

\author{
Hari C. Ghimire and Sean C. C. Bailey* \\ Department of Mechanical Engineering, University of Kentucky, Lexington, Kentucky 40506, USA
}

(Received 6 December 2017; published 16 March 2018)

\begin{abstract}
Time-resolved stereoscopic particle image velocimetry measurements were conducted of a wing-tip vortex decaying in free-stream turbulence. The objective of the research was to experimentally investigate the mechanism causing the increased rate of decay of the vortex in the presence of turbulence. It was observed that the circulation of the vortex core experienced periods of rapid loss and recovery when immersed in free-stream turbulence. These events were not observed when the vortex was in a laminar free stream. A connection was made between these events and distortion of the vortex, coinciding with stripping of core fluid from the vortex core. Specifically, vortex stripping events were connected to asymmetry in the vortex core, and this asymmetry was associated with instances of rapid circulation loss. The increased rate of decay of the vortex in turbulence coincided with the formation of secondary vortical structures which wrapped azimuthally around the primary vortex.
\end{abstract}

DOI: 10.1103/PhysRevFluids.3.034702

\section{INTRODUCTION}

In general, an isolated vortex free of intrinsic instabilities will decay slowly in time, subject only to viscous diffusion. However, its rate of decay can be modified by external influences imposed through the free-stream conditions, and a vortex will therefore experience a greatly increased rate of decay within turbulent surroundings. Interestingly, this process is not well represented by eddy viscosity models, as the increased decay does not occur in the form of an enhanced rate of diffusion.

Instead, the increase in decay rate occurs only through decay of the tangential velocity within the core of the vortex, which outpaces the corresponding rate of increase of core radius $[1,2]$. The core radius remains unaffected by either the presence of, or the intensity of, external turbulence; its rate of growth is unchanged from that produced in a laminar free-stream through viscous diffusion. The increased rate of decay of the vortex, as measured through its circulation, is thus caused by the decrease in tangential velocity in the vortex core, which accounts for almost all the circulation loss when external turbulence is present [2]. As this enhanced decay cannot be attributed to small-scale turbulent diffusion, it must therefore be caused by a more dynamic, three-dimensional process.

Secondary structures in the form of small-scale vortical structures have been observed forming around line vortices immersed in turbulent surroundings in both experiments [2-6] and simulations [7-11]. These secondary structures often form in pairs having alternating signs of azimuthal vorticity [7,9-11] and typically form at a radial distance from the vortex axis of twice the vortex core radius. Once developed, they have a longitudinal scale of approximately the same order of magnitude as the primary vortex core radius [11].

These secondary structures evolve from turbulent eddies that are in close proximity to the primary vortex and become wrapped around the vortex, stretching and intensifying as they do. Once formed,

*sean.bailey@uky.edu 
the secondary structures interact with the primary vortex through different mechanisms. For example, the primary vortex transfers rotational energy to the turbulent eddies while tilting and stretching them in the azimuthal direction during their initial formation [7]. When the strength of the secondary structures is small relative to the primary vortex, they can induce bending waves on to the primary vortex $[9,11,12]$. If the relative intensity is sufficiently high, they can cause the ejection of packets of vorticity from the core of the primary vortex, a process referred to as vortex stripping $[5,6,12]$. Note that the three-dimensional vortex stripping in this context is unrelated to the two-dimensional process examined by Legras and Dritschel [13] and Mariotti et al. [14], who originally coined the term.

Three-dimensional vortex stripping is characterized by high local straining in the radial direction, believed to be induced by the rotation of secondary structures $[8,12]$, and is consistent with observations made by Bandyopadhyay et al. [3]. They observed the intermittent exchange of fluid between the vortex core and the external fluid in flow visualizations. Sufficiently strong vortex stripping can lead to vortex destruction, as has also been observed intermittently in flow visualizations $[5,6]$. The vortex stripping process has thus been studied experimentally, so far, only through qualitative flow visualizations [3,5,6], with most evidence of this process arising from numerical simulations [7-11].

The present work is an extension of the study presented in Ghimire and Bailey [2]. In the previous study, we examined the impact of the turbulence on the mean decay of the vortex, with corresponding observations of nondiffusive decay in the presence of free-stream turbulence, as detailed above. Noting that a mechanism for this nondiffusive decay could be connected to prior observations of an increase in the frequency of vortex stripping when a vortex is within in free-stream turbulence, the objective of the current study is to experimentally investigate and connect the time-dependent decay of the vortex with the presence of vortex stripping. To do this, time-resolved experiments were carried out in which a tip vortex was immersed in isotropic and homogenous turbulence, with measurements of velocity and vorticity field evolution made using time-resolved stereoscopic particle image velocimetry (PIV).

\section{EXPERIMENTAL ARRANGEMENT}

The experiment was performed in a water-filled towing tank of length $3.96 \mathrm{~m}$ and a cross section of $0.43 \times 0.38 \mathrm{~m}^{2}$ (width $\times$ depth). A wing-tip vortex was produced in the tank by towing a finite-span wing and turbulence of different turbulent kinetic energy of different length scales introduced into the tank by towing different grids of different mesh size upstream of the wing. The flow field was interrogated using high-speed stereoscopic time-resolved PIV, allowing the determination of three components of velocity. The coordinate system was aligned with $x$ in the towing direction, $y$ towards the wing root, and $z$ in the direction of lift. The PIV system therefore measured $U_{x}(y, z, t), U_{y}(y, z, t)$, and $U_{z}(y, z, t)$ at a specific $y z$ plane. The overall geometry is presented in Fig. 1. The towing tank, wing, and grids used in this experiment are the same as those used in Ghimire and Bailey [2] with further details are provided below.

The wing used to generate the wing-tip vortex was a NACA0012 finite-span wing having a square tip and rectangular planform of chord length, $C=10 \mathrm{~cm}$. Only $17.8 \mathrm{~cm}$ of the total wing span of $38.1 \mathrm{~cm}$ was submerged within water, with the tip of the wing positioned near the centerline of the measurement plane at a distance of $15.24 \mathrm{~cm}$ from the bottom of the tank. The leading edge of the wing on the suction side was equipped with the boundary layer trip made of sandpaper in order to minimize the formation of leading edge vortex. The wing was set at an $8^{\circ}$ angle of attack, which was determined through flow visualization to be below the stall angle.

To introduce decaying approximately homogeneous and isotropic turbulence, a grid was towed upstream of the wing. Two grids, both having square mesh and solidity ratio of 0.43 but differing in mesh size $M$, were used to produce turbulence with different turbulent kinetic energy and integral length scales. The first, referred to as the "small-grid case," had a mesh size $M=25.4 \mathrm{~mm}$, and the second, referred to as the "large-grid case," had mesh size of $M=35.6 \mathrm{~mm}$. Measurements 


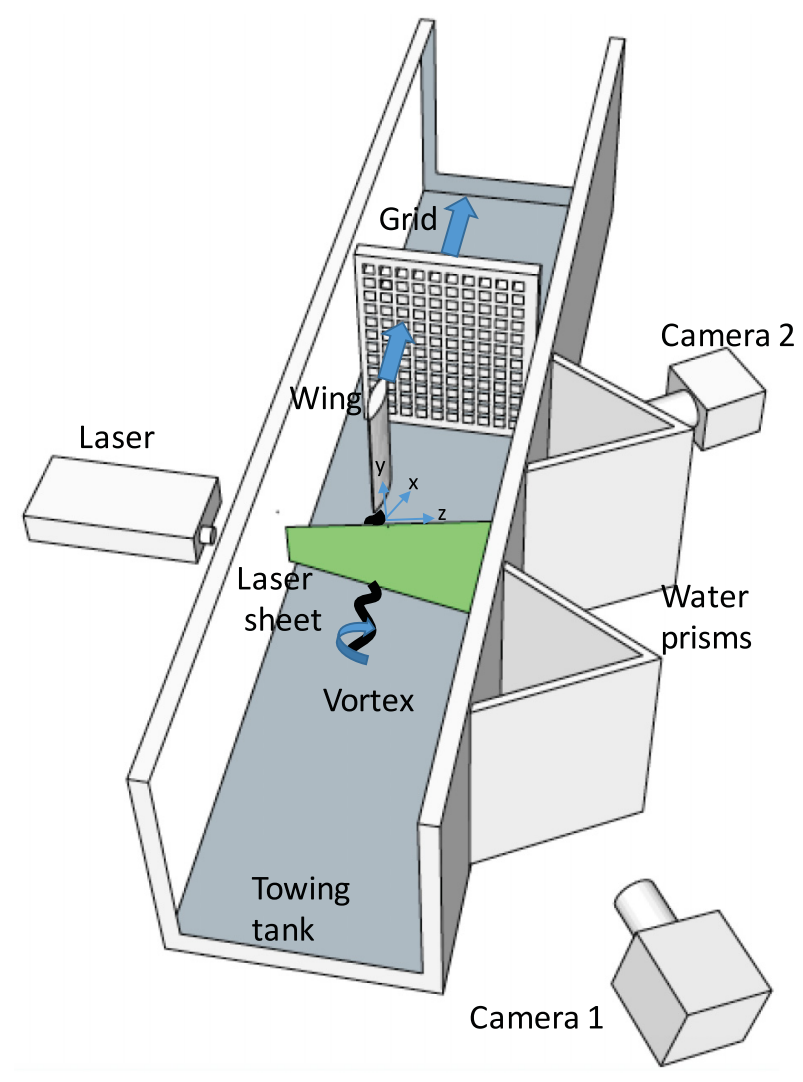

FIG. 1. Experiment configuration.

without the grid will be referred to as "no-grid" case. For the measurement time under consideration the turbulence intensity decays logarithmically from $9 \%$ to $2.2 \%$ for the large grid case and from $7.5 \%$ to $1.75 \%$ for the small-grid case. Further details regarding the characteristics of the turbulence produced by these grids are provided in Ghimire and Bailey [2].

The wing and the grid, positioned $38.1 \mathrm{~cm}$ upstream of the wing, were towed at a constant velocity $U_{t}=0.17 \mathrm{~m} / \mathrm{s}$. This corresponds to a chord-based Reynolds number of $U_{t} C / v=17,000$, where $v$ is the kinematic viscosity.

The effect of surface waves on the vortex evolution was minimized by the relatively low towing speed. However, following Ref. [15], in order to dissipate any such surface waves formed in the towing tank, two vertically placed grids of mesh size $3 \mathrm{~cm}$ and $1.5 \mathrm{~cm}$ separated by $7 \mathrm{~cm}$ distance were placed at a distance of $235 \mathrm{~cm}$ from the measurement plane and $12 \mathrm{~cm}$ from the end of the towing tank. Motion from the measurement plane to the stopping position required nearly $U_{t} t / C=23$. Thus the wing was in constant velocity motion for the duration of the PIV measurement, and the deceleration of the wing is not expected to impact the measurements.

High-speed time-resolved stereoscopic particle image velocimetry was used to obtain threecomponents of the velocity field in a plane normal to the towing direction at a distance approximately the midpoint of the towing tank. The PIV system was composed of a laser, two digital charged coupled device (CCD) cameras with lenses, Scheimpflug mounts, and an external controller.

A Litron LDY302 model laser was used to produce a $527 \mathrm{~nm}$ wavelength light sheet by passing the beam through light sheet optics. For the laser pulse separation and $U_{t}$ used, it was estimated that the laser light sheet thickness of nearly $3 \mathrm{~mm}$ was sufficient to prevent out-of-plane loss of particles from 
the measurement plane. Glass spheres having a density of $1.1 \mathrm{~g} / \mathrm{mL}$ at $25^{\circ}$ centigrade and diameter 9-13 $\mu \mathrm{m}$ were used to seed the water in the towing tank. The seed particles were thoroughly mixed in water and allowed to return to rest before each measurement run. Particle images were captured with two Phantom Miro M310 high-speed digital cameras. The image sensor in the cameras had a resolution of $1280 \times 800$ pixel $^{2}$, pixel size of $20 \mu \mathrm{m}$, and 12-bit pixel depth. The cameras were each equipped with a AF-S VR Micro-Nikkor lens having a focal length $200 \mathrm{~mm}$ and $f$-stop set to 8 . The entire PIV system was controlled by a LaVision high speed controller. Stereoscopic images were taken with these cameras at $160 \mathrm{~Hz}$ for $8.1 \mathrm{~s}$ following the wing crossing the measurement plane.

In order to minimize the effect of refraction, two water-filled prisms were attached to the side wall of the towing tank as shown in Fig. 1. The two cameras were placed approximately normal to the side wall of the prism after adjusting the Scheimpflug adapter to obtain the Scheimpflug criterion of the lens plane and the object plane for each camera intersecting at a common line. The cameras were positioned to receive forward-scattered light from the particles in order to increase the signal-to-noise ratio.

The image pairs were analyzed for $t>0$, where $t=0$ corresponds to the instant of time at which trailing edge of wing just exited the laser sheet plane. LaVision DAVIS 8.1 software was used for processing the particle images. Interrogation region sizes of $24 \times 24$ pixel $^{2}$ and two-pass adaptive cross-correlation were applied with 50\% overlap. Following Keane and Adrian [16], this interrogation area size was chosen so as to have at least 15 particles per interrogation area. After post-processing, $105 \times 80$ vectors with spatial resolution of $\Delta y=\Delta z=1.9 \mathrm{~mm}$ were obtained. This corresponds to between approximately $6 \%$ and $13 \%$ of the vortex core diameter, depending on the distance downstream of the wing. The resulting vector fields were vetted based upon correlation value, allowable velocity range, and comparison of nearest neighbors with less than $2 \%$ of the vectors rejected and then replaced by interpolation.

As done in Ghimire and Bailey [2], the center of the vortex was found with the assumption that the axial vorticity $\Omega_{x}(y, z, t)=\partial U_{z}(y, z, t) / \partial y-\partial U_{y}(y, z, t) / \partial z$ follows binormal or Gaussian distribution [11] such that

$$
\Omega_{x}(y, z, t) \approx B e^{-\left(\frac{(y-\bar{y})^{2}}{2 \sigma_{y}^{2}}-\frac{(z-\bar{z})^{2}}{2 \sigma_{z}^{2}}\right)} .
$$

The optimum fit of Eq. (1) provided an estimate of center position of the vortex $\bar{y}, \bar{z}$, the spatial extent of $\Omega_{x}$ through $\sigma_{y}$ and $\sigma_{z}$, and amplitude of axial vorticity through $B$. The coordinate system was realigned at each measured instant in time such that the origin of the coordinate system was at $\bar{y}$ and $\bar{z}$. Ensemble averaged statistics will be denoted by \langle\rangle , and were found after recentering the coordinate of the vortex axis by averaging 10 runs of data at each instant of time relative to the wing passing through the measurement plane at $t=0$.

\section{RESULTS AND ANALYSIS}

To provide an overview as to how the vortex is impacted by the presence of turbulence, we first examine the rate of decay of the vortex core under different free-stream conditions. The circulation in the vortex core is given by $\Gamma_{c}=2 \pi r_{c} U_{c}$, where $U_{c}$ is the maximum tangential velocity of the vortex and $r_{c}$ is the radius at which it occurs. Note that in this paper we will use a subscripted $c$ to indicate quantities associated with the vortex core, defined as the region $r<r_{c}$ where $r$ is the radial distance from the instantaneous vortex center. Figure 2(a) shows the comparison of ensembleaveraged core circulation $\left\langle\Gamma_{c}\left(\left\langle r_{c}\right\rangle, t\right)\right\rangle /\left\langle\Gamma_{0}\right\rangle$ for no-grid, small-grid, and large-grid cases. To account for small deviations in initial conditions of the vortex introduced by the impact of the free-stream turbulence on the vortex generation process, the core circulation has been normalized by the ensemble averaged total circulation of the vortex, $\left\langle\Gamma_{0}\right\rangle$, determined from the tangential velocity measured at position $r=0.3 C$ at time $t=0$. The increased rate of decay of the vortex core with increasing levels of free-stream turbulence is clearly evident in Fig. 2(a), and is consistent with observations made in previous studies $[1-3,5,6]$. In the present experiments, the no-grid case shows relatively no decay 

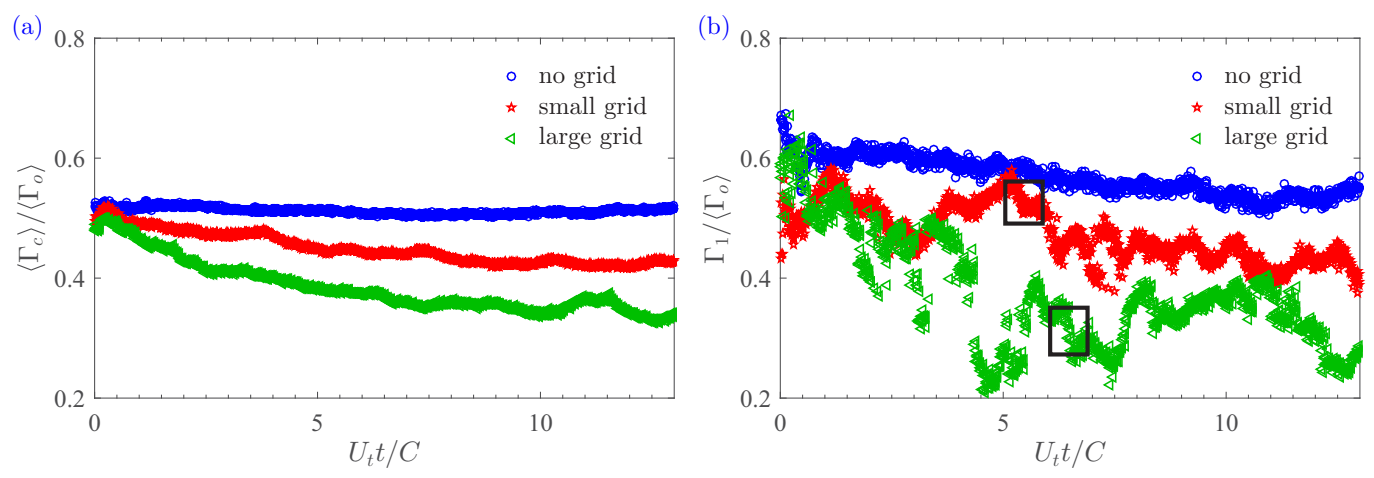

FIG. 2. Comparison of (a) ensemble-averaged core circulation $\left\langle\Gamma_{c}\right\rangle /\left\langle\Gamma_{0}\right\rangle$ and (b) inner circulation $\Gamma_{1} /\left\langle\Gamma_{0}\right\rangle$ for the no-grid, small-grid, and large-grid cases.

in time, whereas the small-grid and large-grid cases have decreased in circulation by $20 \%$ and $50 \%$ respectively by the end of the measurement. For the most part this decay appears to occur gradually and monotonically in time.

The properties of this ensemble-averaged decay were examined in detail in Ref. [2] using experiments conducted at a lower sample rate for a longer time period. It was found that the mean core radius grows from $0.07 C$ to $0.15 C$ by $U_{t} t / C=30$, with the same growth rate observed for all three cases. However, by $U_{t} t / C=10$ the peak tangential velocity for the large-grid case had decreased by $\approx 50 \%$, whereas over the same distance it had only decreased by $\approx 65 \%$ for the small-grid case and $75 \%$ for the no-grid case. The net result was a corresponding loss in circulation, very similar to that observed in the present experiments. In Ref. [2], it was also observed that this ensemble-averaged loss of circulation is almost completely due to loss of vorticity from the vortex core.

Although the vortex decay on average appears gradual, it was observed that the decay process for a single member of the ensemble was much more dynamic. However, due to unsteady asymmetric deformation of the vortex core when immersed in turbulence, $r_{c}$ and $U_{c}$ are poorly defined quantities in an instantaneous velocity field. We therefore used the swirling strength, $\lambda$, to identify the core of the vortex in each individual PIV frame. The swirling strength was found from the normal strain $E_{y y}, E_{z z}$ and shear strain $E_{y z}, E_{z y}$ in the $y z$ plane [17], following

$$
\lambda=-E_{y z} E_{z y}+\frac{E_{y y} E_{z z}}{2}-\frac{E_{y y}^{2} E_{z z}^{2}}{4}
$$

such that when the value of $\lambda$ is negative the velocity field is in shear, and when the value of $\lambda$ is positive it is in swirl. Thus by thresholding $\lambda$ above a certain value, we can identify the region of high swirl associated with the primary vortex while neglecting regions of shear which exist in the wing wake and turbulence. Specifically, to identify regions of the core $\lambda(y, z, t)$ has been thresholded by defining $\lambda_{c}$ as a suitable threshold. We can then define $\Gamma_{1}(t)$ by integrating the vorticity over the region where $\lambda>\lambda_{c}$ such that

$$
\Gamma_{1}(t)=\int_{\lambda>\lambda_{c}} \Omega_{x}(y, z, t) d y d z
$$

Note that $\lambda_{c}$ was selected to achieve the greatest parity between $\left\langle\Gamma_{1}\right\rangle$ and $\left\langle\Gamma_{c}\right\rangle$.

A comparison of $\Gamma_{1}(t) /\left\langle\Gamma_{0}\right\rangle$ between the no-grid, small-grid, and large-grid cases for a single member of the ensemble, i.e., a single towing run, is presented in Fig. 2(b). As with the ensembleaveraged decay of $\left\langle\Gamma_{c}\right\rangle$, the overall rate of decay of $\Gamma_{1}$ was found to be higher for the cases with the free-stream turbulence than the baseline no-grid case over the entire measurement time. Also, as with $\left\langle\Gamma_{c}\right\rangle$, the evolution of $\Gamma_{1}$ for the no-grid case was approximately smooth and relatively constant in time, reflecting slow, approximately constant decay. Conversely for the cases with free-stream 

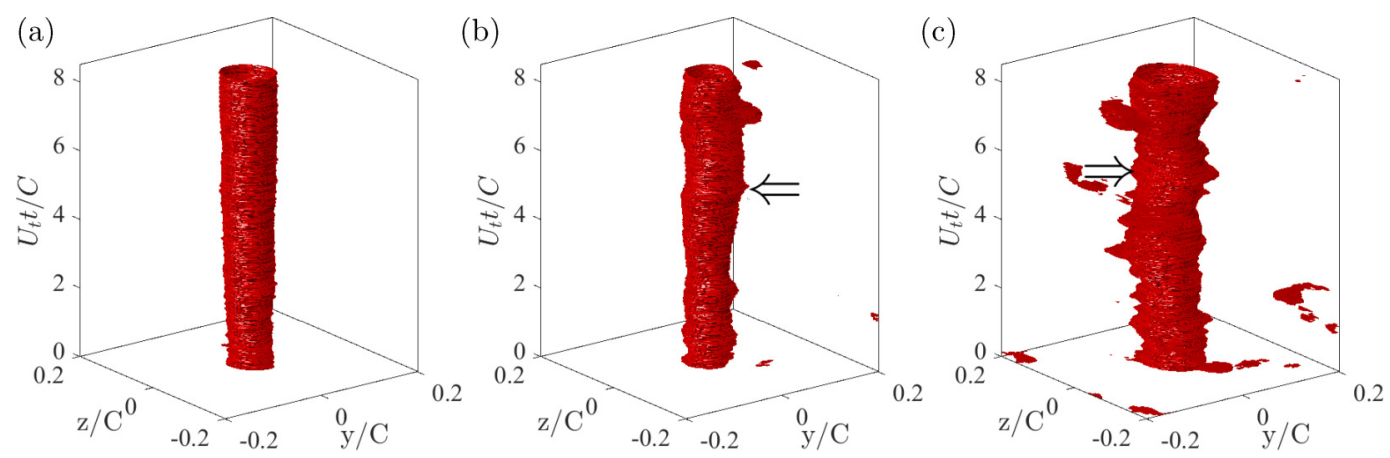

FIG. 3. Isosurfaces showing evolution of $U_{\theta} r /\left\langle\Gamma_{0}\right\rangle=0.035$ with $U_{t} t / C$ for a single member of the ensemble of the (a) no-grid, (b) small-grid, and (c) large-grid cases.

turbulence, although there is overall decay evident, the decay of $\Gamma_{1}$ is marked by abrupt changes in the form of both positive and negative gradients of $\Gamma_{1}$. To illustrate, a single event of rapid drop followed by rapid increase has been indicated for both the small-grid and large-grid cases by a box in Fig. 2(b). These specific events will be examined in more detail later in this paper and associated with instances of vorticity ejection from the core, i.e., vortex stripping.

The structure of these changes in circulation can be visualized through isosurfaces of $U_{\theta} r$, where $U_{\theta}(r, t)$ represents the tangential velocity. Thus, $U_{\theta} r$ constitutes a representation of what the local contribution to the vortex strength would be if the vortex were axisymmetric [e.g., assuming $\Gamma(r)=$ $2 \pi r U_{\theta}$ excluding the constant value term $\left.2 \pi\right]$. Isosurface plots of the time evolution of $U_{\theta} r /\left\langle\Gamma_{0}\right\rangle=$ 0.035 are presented for the no-grid, small-grid, and large-grid cases in Figs. 3(a)-3(c). For the no-grid case, the iso-surfaces are axisymmetric, representing the axisymmetry of the vortex in this case, with a slight growth in time corresponding to the decay in no-grid case circulation observed in Fig. 2. Unlike the no-grid case, however, the isosurfaces of $U_{\theta} r$ for the grid cases were characterized by protrusions appearing irregularly in time. These bulges indicate a localized deviation from axisymmetry in the vortex and correspond to the abrupt changes in $\Gamma_{1}$ in Fig. 2(b). For example, the instances highlighted in Fig. 2(b) have been indicated by an arrow in Figs. 3(b) and 3(c). At these instances, a protrusion in $U_{\theta} r$ occurs. When the same instances are viewed on the circulation evolution, it can be observed that they correspond to the net circulation experiencing a local minimum. In both cases, the recovered magnitude of vortex circulation following the local minimum is lower than before it occurred.

An alternate illustration of the structure of these protrusions is provided by the sectional view at constant $\theta$ of $U_{\theta} r$ for different $t$, as presented in Figs. 4(a)-4(c) for the no-grid, small-grid, and large
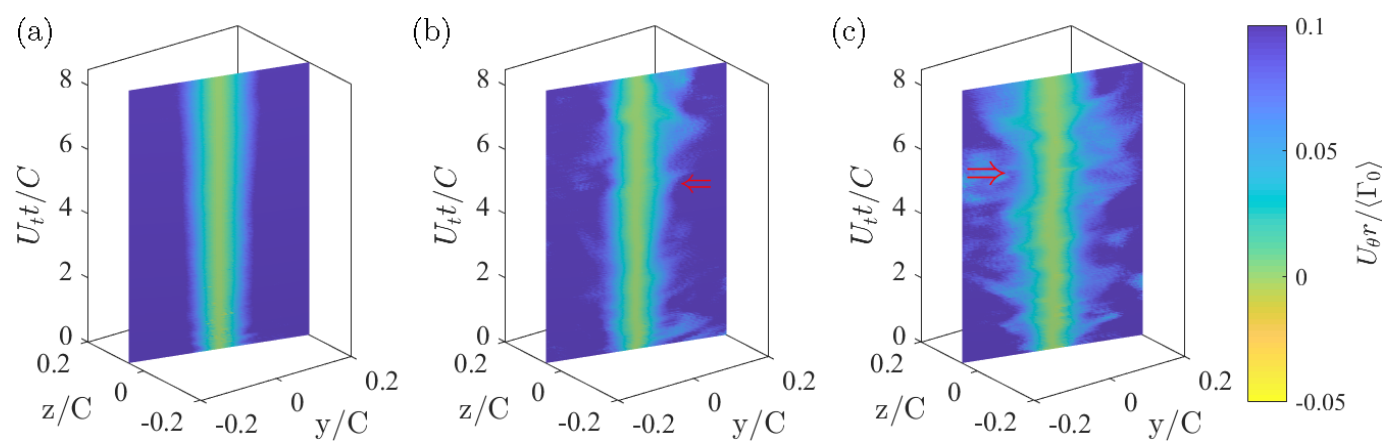

FIG. 4. Contour plots at constant angle of $U_{\theta} r /\left\langle\Gamma_{0}\right\rangle$ showing its evolution with $U_{t} t / C$ for a single member of the ensemble taken during measurement of the (a) no-grid, (b) small-grid, and (c) large-grid cases. 

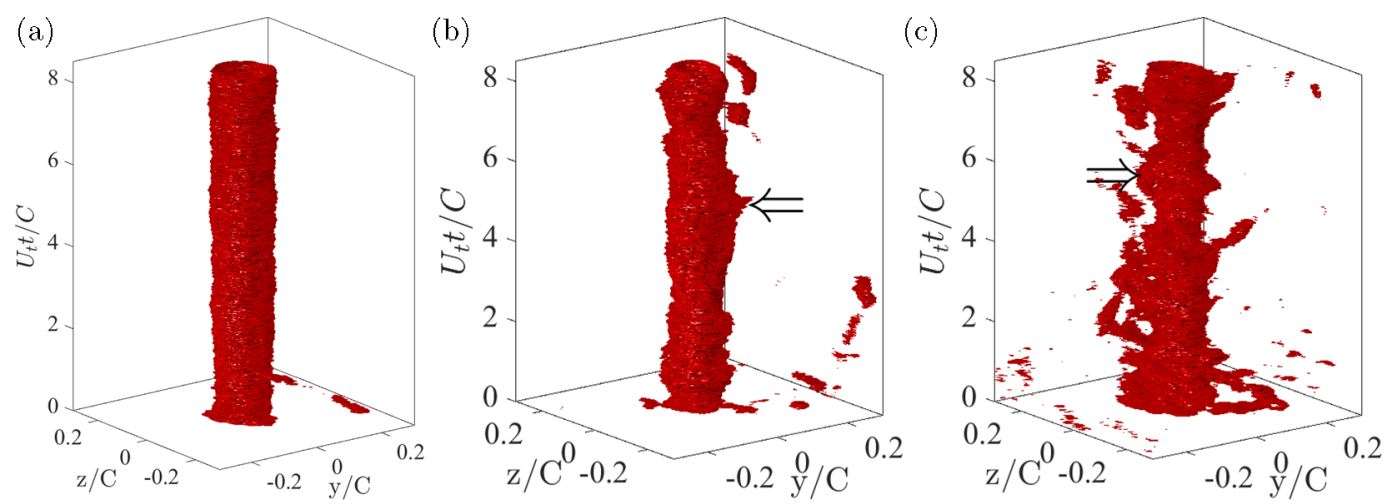

FIG. 5. Isosurface plots of normalized swirling strength $\lambda /\left\langle\Omega_{x}^{\star}\right\rangle^{2}=0.008$ showing its evolution with $U_{t} t / C$ for a single member of the ensemble taken from measurements of (a) no-grid, (b) small-grid, and (c) large-grid cases.

grid cases. The angle of the sectional plane was specifically selected to highlight as many of these events as possible. The no-grid case showed slow growth of $U_{\theta} r$, reflecting the gradual decay of $\Gamma$. However, the protrusions observed in Figs. 3(b) and 3(c) for the two grid cases take the appearance of "ejections," suggesting instances where core fluid is discharged from the core of the vortex into the free stream. To highlight the connection to protrusions observed above, the same protrusions indicated in Figs. 3(b) and 3(c) for the grid cases have once again been marked in Figs. 4(b) and 4(c).

Thus, it appears that there are local deviations in core axisymmetry which correspond to changes in circulation, and the structure of these deviations suggests ejection of core fluid into the free stream, reminiscent of a vortex stripping event. To establish a better connection between these events and vortex stripping, the vortex swirling strength $\lambda$ can be revisited, as the majority of the swirling strength is contained within the vortex core and ejections of this fluid should maintain some swirling strength once ejected. Isosurface plots of the time evolution of swirling strength $\lambda /\left\langle\Omega_{x}^{\star}\right\rangle^{2}=0.008$ for the no-grid, small-grid, and large-grid cases are shown in Figs. 5(a)-5(c). The swirling strength has been normalized by the maximum measured ensemble-averaged peak axial vorticity of the vortex, $\left\langle\Omega_{x}^{\star}\right\rangle$, to account for small deviations in initial conditions of the vortex introduced by the impact of the free-stream turbulence on the vortex generation process.

The slow diffusion of the vortex in the absence of free-stream turbulence is evident in the axisymmetry of the $\lambda$ isosurface of the no-grid case shown in Fig. 5(a). However, the protrusions present in the iso-surface plots of $U_{\theta} r$ observed for the grid cases have corresponding protrusions in the iso-surfaces of $\lambda$. This is highlighted by the arrow which shows the same instance in time indicated in Figs. 2(b), 3(b), 3(c), 4(b), and 4(c). The isosurfaces of $\lambda$ reveal that these protrusions appear as arms of strong swirling fluid radiating outward from the primary vortex, with these arms consistent with core fluid ejecting into the free stream.

Further details of the evolution of these events can be found in the contours of the evolution of $\lambda$ in time provided in Figs. 6(a)-6(c). As before, the angle of the plane shown was specifically selected to highlight as many of these events as possible. The nogrid case was marked by gradual decrease in $\lambda$ along constant $r$. However, the grid cases display instances at which protrusions of high swirling strength move away from the vortex in time, characteristic of vortex stripping, that are not present for the no-grid case. Again, the same instances in time marked in Fig. 2(b) have been highlighted in Figs. 6(b) and 6(c).

That these protrusions correspond to vortex stripping can be further verified using the contour plots of $\lambda(y, z)$ provided in Figs. 7(a)-7(i), which cover a time interval of $\Delta t=0.021 C / U_{t}$ for (a)-(c) no-grid, (d)-(f) small-grid, and (g)-(i) large-grid cases. For the no-grid case, the contours of $\lambda$ are approximately axisymmetric and show little change in time. When immersed in turbulence, as 

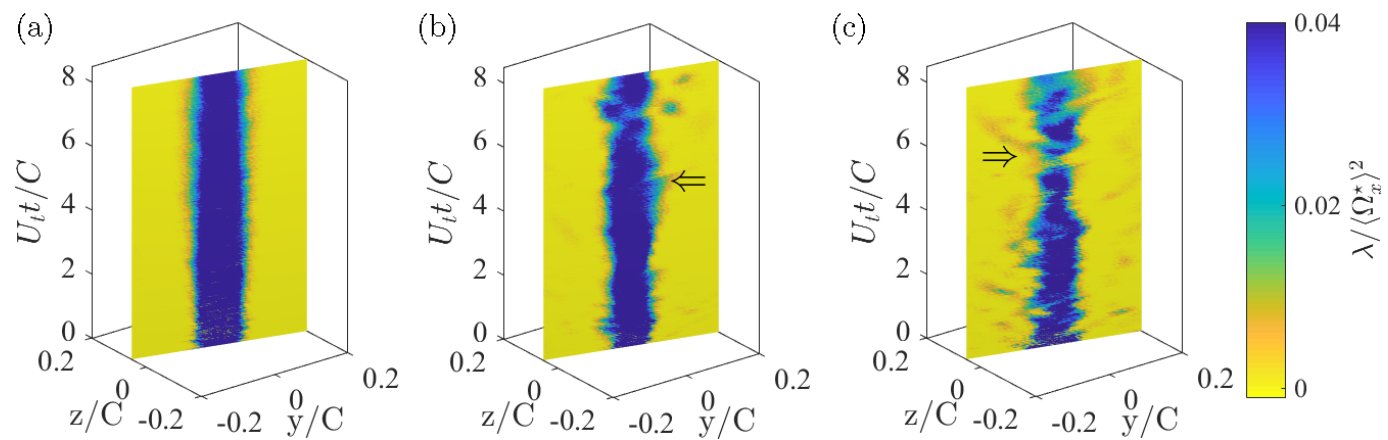

FIG. 6. Contour plots at constant angle showing the change in $\lambda /\left\langle\Omega_{x}^{\star}\right\rangle^{2}$ with $U_{t} t / C$ for a single member of the ensemble taken from measurements of (a) no-grid, (b) small-grid, and (c) large-grid cases.

can be observed in Figs. 7(d)-7(f) and 7(g)-7(i), the vortex is no longer axisymmetric but evolves dynamically due to straining of the vortex by the turbulence. Specifically, protrusions of high swirl can be observed emitting from the core in Fig. 7(e) for the small-grid case and Fig. 7(g) for the large-grid case. These protrusions become cut off from the core in Figs. 7(f) and 7(h), forming isolated regions of high swirl which then decay rapidly [e.g., as shown by comparison of Figs. 7(h) to 7(i)]. These figures are representative examples of a process which occurred frequently in the data and illustrates the stripping of highly vortical fluid from the vortex core.

As noted, the vortex stripping process coexists with asymmetry in the vortex core. To relate this process to the loss of circulation from the vortex, we compare the rate of change of the inner circulation, $d \Gamma_{1} / d t$, with the circularity of the vortex. Recall that $\Gamma_{1}$ is the circulation calculated for an area, $A_{1}$, for which the value of $\lambda$ exceeded a certain threshold. We therefore define the circularity
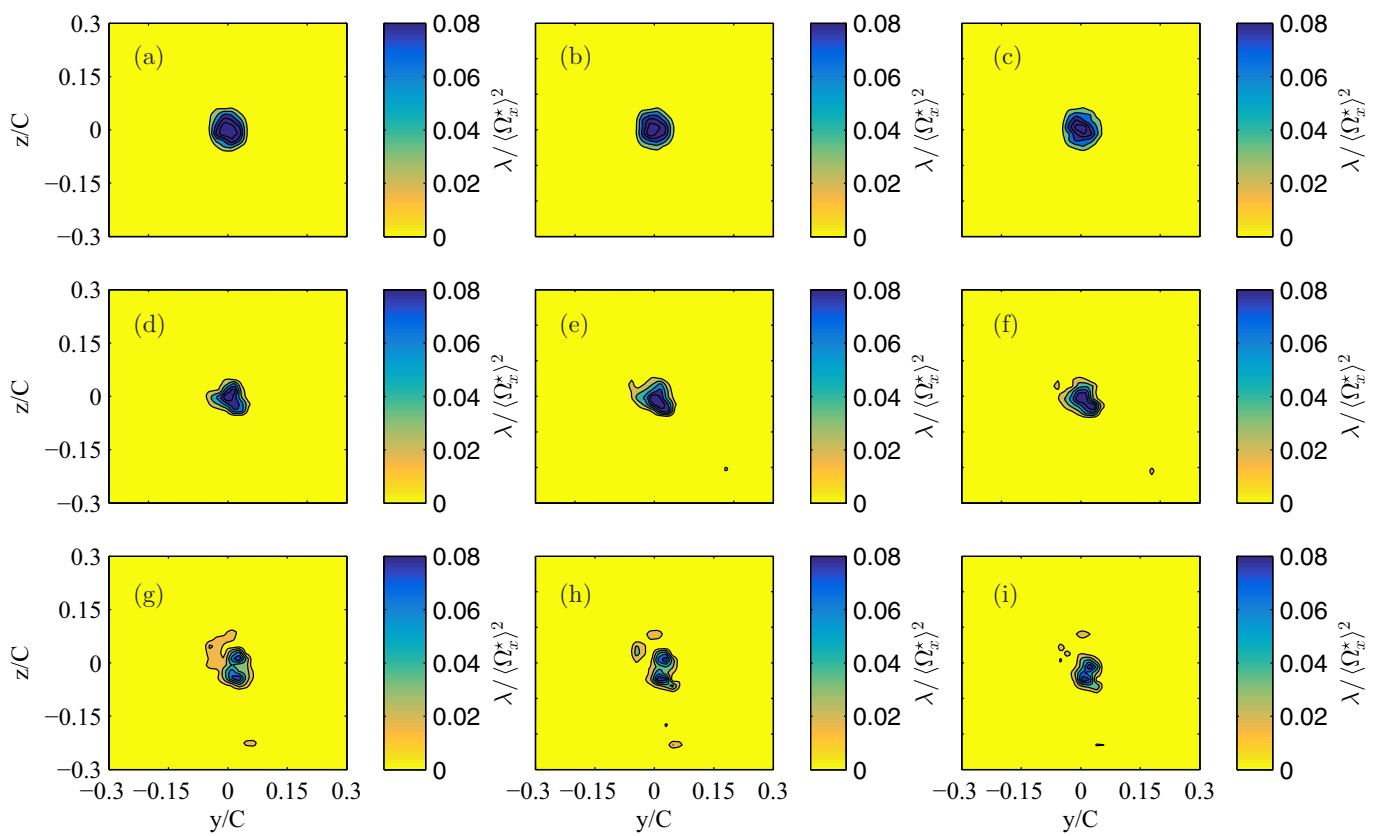

FIG. 7. Contour plots of swirling strength $\lambda /\left\langle\Omega_{x}^{\star}\right\rangle^{2}$ covering time interval $\Delta t=0.021 C / U_{t}$ for (a)-(c) no-grid, (d)-(f) small-grid, and (g)-(i) large-grid cases with the initial at $U_{t} t / C=1.02$. 

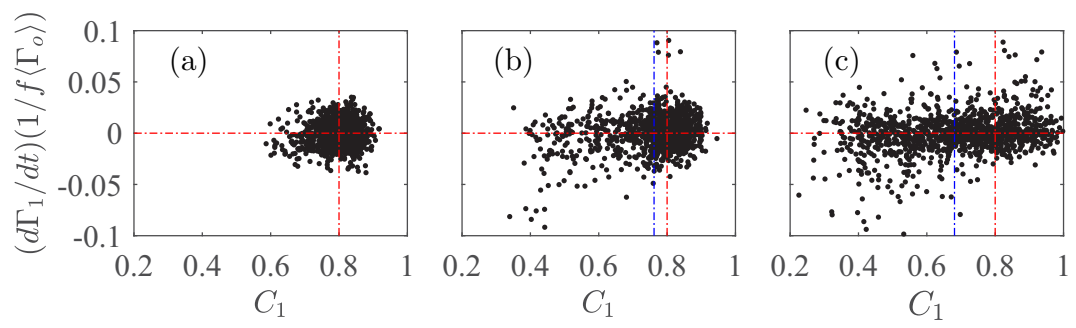

FIG. 8. Scatter plots of normalized $d \Gamma_{1} / d t$ plotted against $C_{1}$ for (a) no-grid, (b) small-grid, and (c) largegrid cases. Dashed red lines indicate the mean value of $C_{1}$ for the no-grid case and zero level of circulation decay. Dashed blue lines in (b) and (c) indicate the mean value of $C_{1}$ for small-grid and large-grid cases.

as $C_{1}=\left(4 \pi A_{1}\right) / P_{1}^{2}$, where $P_{1}$ is the length of the perimeter of $A_{1}$. Thus a value of $C_{1}=1$ indicates a perfectly axisymmetric vortex.

The rate of change of circulation and circularity are compared for the no-grid and grid cases in the form of scatter plots in Figs. 8(a)-8(c). In none of the cases was the vortex perfectly axysymmetric, with the baseline no-grid case having a measured circularity centered around $C_{1}=0.8$, and the rate of change of circulation is centered near zero. In addition, experiencing a reduction in the mean of the vortex circularity, which increases with the turbulence intensity, the two grid cases also show a much greater variability in $C_{1}$. Although the varying circularity is not always associated with a loss or gain of circulation, the instances where strong vortex decay occurs predominantly coincides with instances when $C_{1}$ is below the mean value of 0.8 observed for the no-grid case. Instances where circulation recovery occurs tends to be associated with a wide range of $C_{1}$, but at generally higher values than where circulation is lost. When $C_{1}>0.8$ there are instances circulation increase, but no evidence of decay. Thus, increased instances of circulation loss introduced by the presence of external turbulence can be attributed to vorticity stripping events, themselves characterized by stretching and deformation of the vortex core.

The above discussion has provided evidence that the additional loss of circulation which occurs when a vortex is immersed in turbulence is associated with ejection of the core fluid ejection into the free-stream. Previous studies [7,11] have related these instances of vortex stripping to secondary structures which form around the vortex. The present experiments are not well suited for drawing a concrete connection between the secondary structures and the vorticity events. However, we can demonstrate an increased prominence of the secondary structures surrounding the primary vortex when free-stream turbulence is present. As these structures form azimuthally around the primary vortex their signature is most evident in the azimuthal component of vorticity,

$$
\Omega_{\theta}=\frac{\partial U_{r}}{\partial x}-\frac{\partial U_{x}}{\partial r}
$$

where $U_{r}$ is the radial component of velocity.

As the current experiments did not allow the measurement of axial gradients, we instead examine the pseudo azimuthal vorticity $\Omega_{\theta \theta}=-\partial U_{x} / \partial r$, i.e., the contribution from the radial gradients of the stream-wise velocity to the azimuthal vorticity. Isosurfaces of pseudo azimuthal vorticity at three levels are presented in Figs. 9(a)-9(c). The three isosurface levels were selected to highlight different features, with the strongest contributions to $U_{\theta \theta}$ coming from axial gradients within the vortex core. However, at lower levels of both positive and negative $U_{\theta \theta}$ the signature of secondary structures becomes evident, appearing as regions of coherent vorticity wrapping around the primary vortex. The structures appear with alternating sign at a distance nearly two to three times the core radius value and are thus consistent with the observations of secondary structures made in previous studies [7,9-11]. For the no-grid case, such structures are not evident. However, as the free-stream turbulence intensity increases the density of the structures increases accordingly. Note that, although 

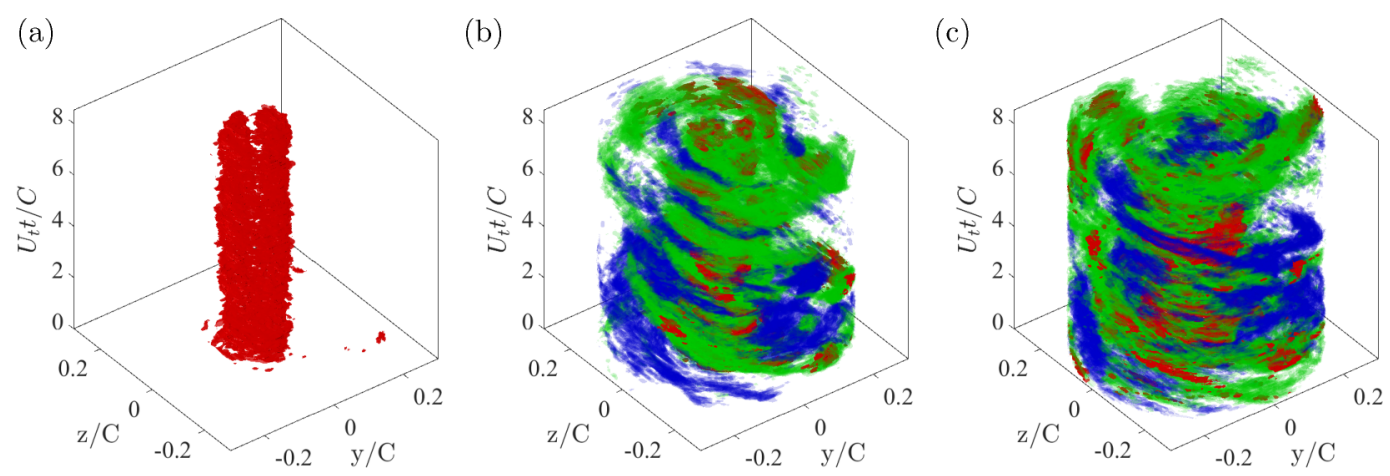

FIG. 9. Isosurface plots of pseudo azimuthal vorticity $\Omega_{\theta \theta} /\left\langle\Omega_{x}^{\star}\right\rangle=0.09$ (red), $\Omega_{\theta \theta} /\left\langle\Omega_{x}^{\star}\right\rangle=0.05$ (green), and $\Omega_{\theta \theta} /\left\langle\Omega_{x}^{\star}\right\rangle=-0.05$ (blue) with $U_{t} t / C$ for (a) no-grid, (b) small-grid, and (c) large-grid cases.

the turbulence intensity is decaying in time, the strength of these azimuthally aligned structures does not, which could reflect their persistence in time.

\section{CONCLUSIONS}

The objective of this study was to experimentally investigate the increased rate of decay of the vortex in the presence of external turbulence. To do this, a wing-tip vortex was generated by towing a NACA0012 wing oriented at $8^{\circ}$ angle of attack, and nearly homogeneous and isotropic turbulence of different kinetic energy and length scales was produced upstream of the wing by towing one of two turbulence-generating grids upstream of the wing. Measurements of velocity and vorticity field time evolution were obtained using time-resolved stereoscopic particle image velocimetry at a fixed plane.

When the vortex was generated within laminar surroundings, the vortex diffused very gradually, and the core experienced little circulation loss. It has previously been observed that a vortex immersed in turbulence will experience circulation decay; however, this decay is nondiffusive, occurring as a loss of azimuthal velocity without a corresponding growth of vortex core size. In the present study, it was found that when the vortex was immersed in turbulent surroundings, the circulation of the vortex core experienced periods of rapid loss, followed immediately by a rapid increase in circulation. However the recovered circulation tended to be lower than it was before the initial circulation loss. These instances were found to coincide with distortion of the vortex core, which itself was characterized by the ejection of swirling fluid from the core, indicative of vortex stripping. By recognizing that these ejection events were characterized by stretching and deformation of the vortex core, and noting that this deformation increased with the turbulence intensity, it was possible to connect the instances of faster decay of the circularity of the vortex and hence connect the sudden loss of circulation to vortex stripping events. Thus, the present study supports the hypothesis that the increased rate of decay of vortex circulation which occurs in turbulent surroundings is a stochastic process characterized by vortex stripping.

Coinciding with the vortex stripping was the formation of secondary, azimuthally aligned vortical structures, evident when the free-stream turbulence was present. The secondary structures were arranged with alternate opposite signs at a radial position corresponding to nearly two to three times the core radius value, and their intensity increased with the turbulence intensity. Previous studies have suggested similar structures produce vortex stripping through generation of intense localized straining. Unfortunately, the nature of the experiments prevented a true measurement of azimuthal vorticity, and hence the relationship between secondary structures and the vortex stripping process could not be examined in detail. 
[1] S. C. C. Bailey and S. Tavoularis, Measurements of the velocity field of a wing-tip vortex, wandering in grid turbulence, J. Fluid Mech. 601, 281 (2008).

[2] H. C. Ghimire and S. C. C. Bailey, An experimental investigation of wing-tip vortex decay in turbulence, Phys. Fluids 29, 037108 (2017).

[3] P. R. Bandyopadhyay, R. L. Ash, and D. J. Stead, Organized nature of a turbulent trailing vortex, AIAA J. 29, 1627 (1991).

[4] M. L. Beninati and J. S. Marshall, An experimental study of the effect of free-stream turbulence on a trailing vortex, Exp. Fluids 38, 244 (2005).

[5] H.-T. Liu, Effects of ambient turbulence on the decay of a trailing vortex wake, J. Aircraft 29, 255 (1992).

[6] T. Sarpkaya and J. J. Daly, Effect of ambient turbulence on trailing vortices, J. Aircraft 24, 399 (1987).

[7] F. Holzäpfel, T. Hofbauer, D. Darracq, H. Moet, F. Garnier, and C. F. Gago, Analysis of wake vortex decay mechanisms in the atmosphere, Aerosp. Sci. Technol. 7, 263 (2003).

[8] J. Marshall and M. Beninati, External turbulence interaction with a columnar vortex, J. Fluid Mech. 540, 221 (2005).

[9] M. V. Melander and F. Hussain, Coupling between a coherent structure and fine-scale turbulence, Phys. Rev. E 48, 2669 (1993).

[10] D. Pradeep and F. Hussain, Vortex dynamics of turbulence-coherent structure interaction, Theor. Comput. Fluid Dyn. 24, 265 (2010).

[11] N. Takahashi, H. Ishii, and T. Miyazaki, The influence of turbulence on a columnar vortex, Phys. Fluids 17, 035105 (2005).

[12] J. Marshall, The flow induced by periodic vortex rings wrapped around a columnar vortex core, J. Fluid Mech. 345, 1 (1997).

[13] B. Legras and D. Dritschel, Vortex stripping and the generation of high vorticity gradients in twodimensional flows, Appl. Sci. Res. 51, 445 (1993).

[14] A. Mariotti, B. Legras, and D. G. Dritschel, Vortex stripping and the erosion of coherent structures in two-dimensional flows, Phys. Fluids 6, 3954 (1994).

[15] K. Hayashi and J. R. Chaplin, Wave damping by an array of circular cylinders and a group of model plants, in Proceedings of the 9th International Symposium on Ecohydraulics, IAHR (University of Natural Resources and Life Sciences, Vienna, Austria, 2012).

[16] R. D. Keane and R. J. Adrian, Optimization of particle image velocimeters. Part I: Double pulse systems, Meas. Sci. Technol. 1, 1202 (1990).

[17] LaVision, DaVis 8.1 software, Product Manual 1003001. 